\title{
FDG PET/ CT detected an insidious inguinal canal metastasis in a patient with pancreatic cancer
}

\author{
Leonard T Ong ${ }^{1}$, Akin Yildiz², Lale Kostakoglu ${ }^{3}$ \\ 1. Department of Radiology, Division of Nuclear Medicine, Memorial Sloan Kettering Cancer Center, New York, United \\ States. 2. Faculty of Medicine, Akdeniz University, Antalya, Turkey. 3. Mount Sinai School of Medicine, New York, United \\ States.
}

Correspondence: Lale Kostakoglu. Address: One Gustave L. Levu Place, Box: 1141, New York NY 10029. Telephone: 212-241-7888. Fax: 212-831-2851. E-mail: lale.kostakoglu@mssm.edu

Received: December 8, $2011 \quad$ Accepted: January 11, $2012 \quad$ Published: June 1, 2012

DOI : 10.5430/jnep.v2n1p96 URL: http://dx.doi.org/10.5430/jnep.v2n1p96

\begin{abstract}
A 70 year old male with a history of inoperable pancreatic cancer with peritoneal metastases was evaluated by FDG PET/CT imaging for disease extent and therapy response after 4 cycles of chemotherapy. PET/CT demonstrated mildly increased uptake at the primary site within the pancreatic neck and in the peritoneum. An unexpected finding involved increased uptake in the right inguinal canal region around the patient's known hernia. A hernia inflammation was suspected and the patient subsequently underwent surgical intervention for possible strangulation. Unexpectedly, the histopathologic evaluation of the surgical specimen revealed peritoneal metastasis in the inguinal canal. Although metastasis to this localization is rather rare, the use of PET/CT assisted in the discovery of unexpected metastasis in a rare location.
\end{abstract}

\section{Key words}

FDG PET/CT, Pancreatic cancer, Inguinal canal, Peritoneal metastases

\section{Introduction}

\section{Case presentation:}

A 70 year old male presents with a history of inoperable pancreatic cancer with peritoneal metastases diagnosed nine months ago. The histology was consistent with moderately differentiated non mucinous pancreatic adenocarcinoma. He underwent 4 cycles of combination chemotherapy between January and June 2009. During therapy, his CEA levels decreased from 365 to 165 , indicating good response to ongoing therapy. Due to severe therapy induced hematologic toxicity, chemotherapy was discontinued and the patient was referred for further testing including serum tumor markers, CT and PET/CT studies. The CT study showed a pancreatic neck mass which decreased in size compared to baseline CT. A subsequent PET/CT revealed a mildly hypermetabolic solid mass within the neck of the pancreas extending into the pancreatic body (SUVmax 4.0) (see Figure 1, arrow). Additionally, there were scattered areas of nodular stranding within the mesentery with several areas demonstrating mild FDG uptake (SUVmax 3.0) (see Figure 1, arrowhead) and an $8 \mathrm{~mm}$ precaval lymph node with mild FDG uptake (not shown). Furthermore, a large right inguinal canal defect was noted to demonstrate soft tissue stranding associated with increased FDG uptake (SUVmax 4.0) (see Figure 2, arrow). Overall, the 
PET/CT findings were consistent with pancreatic cancer post chemotherapy that showed mesenteric stranding with mild FDG uptake. In the absence of a baseline study, the degree of response to therapy cannot be optimally assessed. However, the findings in the inguinal canal were most intriguing which required further evaluation by a surgeon. The patient subsequently underwent inguinal hernia repair with the suspicion of an impending incarcerated hernia based on physical examination. At the time of surgery, there was a hard mass in the right inguinal region emanating from the internal ring. The mass was not typical for incarcerated hernia. Analysis by frozen section and permanent histopathologic sections revealed the mass to be adenocarcinoma, consistent with peritoneal metastasis from patient's known pancreatic adenocarcinoma.
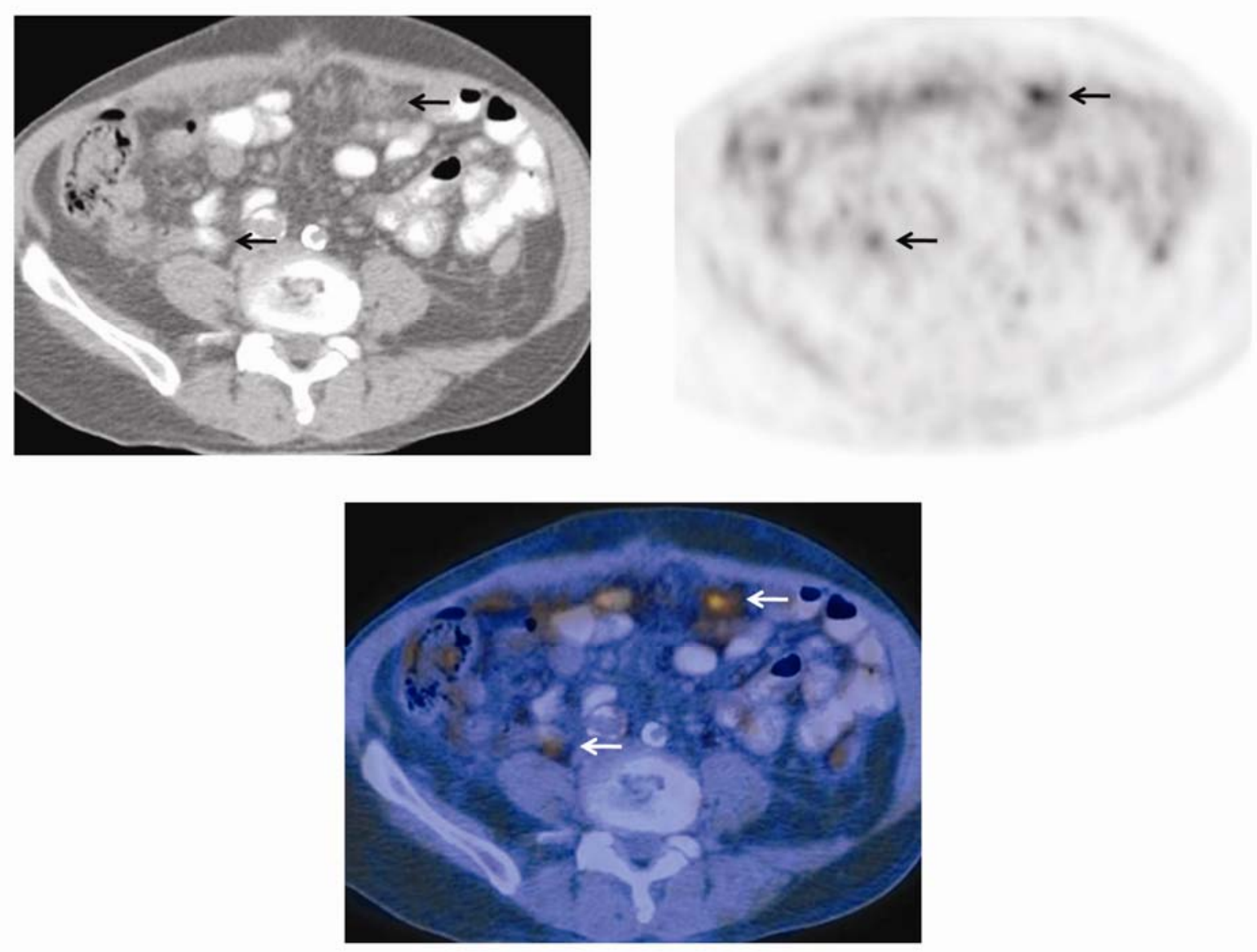

Figure 1. PET/CT revealing scattered areas of nodular stranding within the mesentery with several areas demonstrating mild FDG uptake (SUVmax 3.0) corresponding to peritoneal metastases (arrows). These lesions were noted to have decreased in size when compared with the baseline CT study.

\section{Discussion}

Pancreatic adenocarcinoma (PC) is the fourth leading cause of cancer-related death in the United States ${ }^{[1]}$. By the time patients typically present with pancreatic cancer their prognosis is poor due to advanced staging. The 5-year survival rate is $5 \%{ }^{[2]}$. Approximately $95 \%$ of pancreatic cancers are adenocarcinomas. Surgical resection is potentially curative for pancreatic cancer. However, pancreatectomy can only be done in $15-20 \%$ of presenting patients ${ }^{[3]}$.

PC constitutes a major challenge to the managing physician, both at diagnosis and in the follow up period for detection of residual or recurrent disease, due to its retroperitoneal location and insidious onset. The diagnosis of PC is often made both 
radiographically and histologically ${ }^{[4]}$. Laboratory values may show an increase in serum levels of lipase, bilirubin, and alkaline phosphatase. In a study of 135 subjects using tumor markers CA19-9 and CEA to detect PC, CA19-9 and CEA were shown to have a sensitivity of $80 \%$ and $40 \%$, with a specificity of $67.5 \%$ and $73 \%$, respectively ${ }^{[5,6]}$. Despite developments in MRI and ultrasound, CT remains the modality of choice for the diagnosis, staging, and restaging of PC with an accuracy of $69-94 \%$, and an accuracy of $63-95 \%$ in the detection of recurrence ${ }^{[5,7-9]}$. This data was based upon tumors greater than $2.0 \mathrm{~cm}$; therefore the accuracy of anatomic modalities in the detection of small lesions has yet to be determined. The diagnostic algorithm in the post therapy setting is similar to that used at initial diagnosis and staging. However, morphologic modalities cannot differentiate between post therapy changes from residual disease. More recently, FDG PET/CT has emerged as a powerful diagnostic tool in the evaluation of malignant processes and proven to be superior to existing morphologic modalities, particularly in the detection of recurrent or residual PC. The success of $\mathrm{PET} / \mathrm{CT}$ is mainly due to its ability to detect metabolic changes early during the course of disease, which usually precede morphologic changes. A study by Ruf et al ${ }^{[20]}$ reported a sensitivity and a specificity of $96 \%$ and $100 \%$ for FDG PET/CT in the detection of recurrent disease at various sites including both hepatic and extrahepatic disease, while CT/MRI yielded similar specificity but a sensitivity of only $39 \%$.
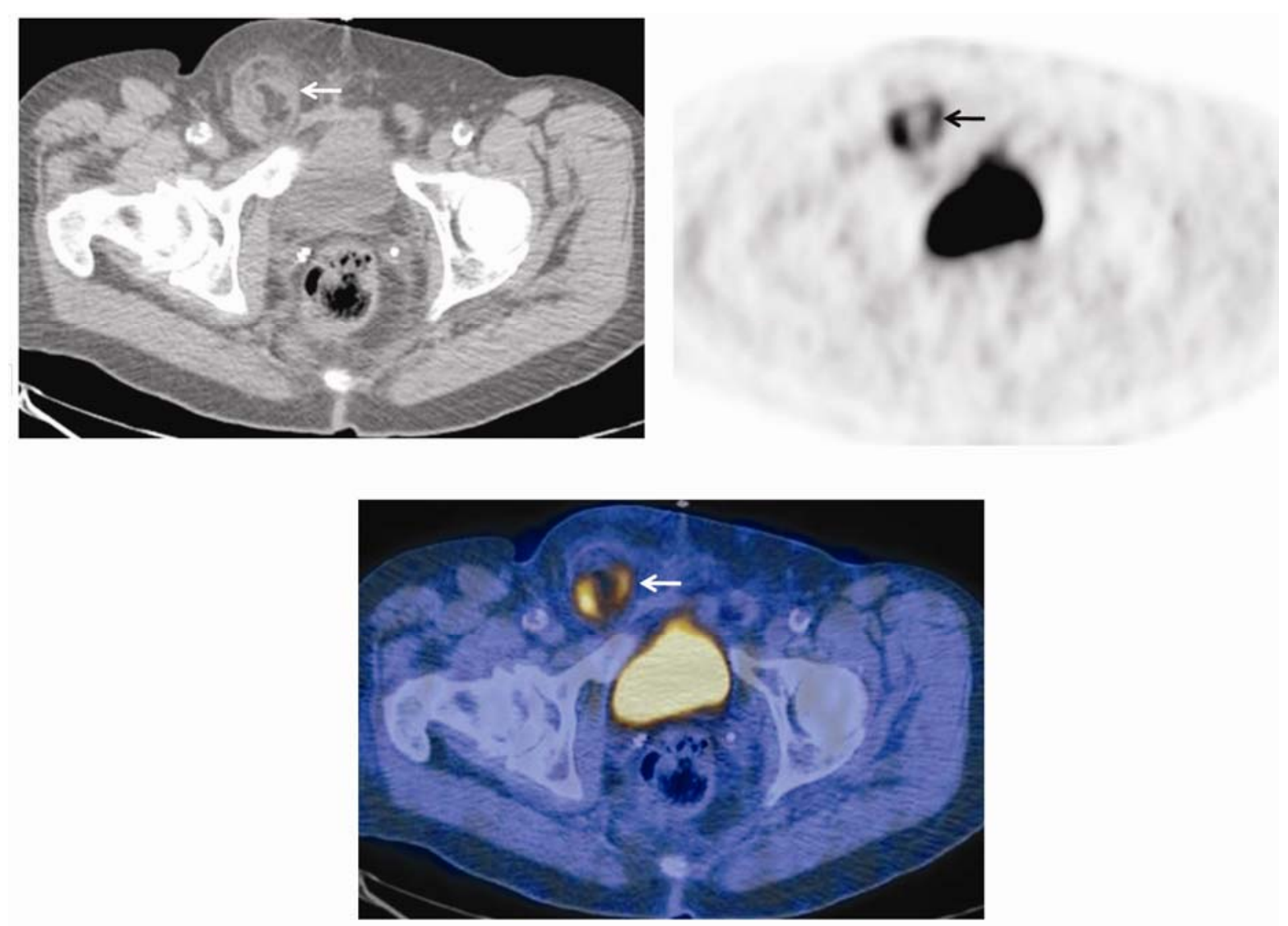

Figure 2. A large right inguinal canal defect is noted to demonstrate soft tissue stranding associated with increased FDG uptake (SUVmax 4.0) (arrows). Histopathologic sections of the mass revealed adenocarcinoma, consistent with metastasis from the patient's known pancreatic primary malignancy

PC metastases can occur via lymphatics or hematogenously, depending on the level of expression of growth-promoting factors within the tumor mass and the disturbance of growth-inhibitory factors ${ }^{[10]}$. Common sites of pancreatic metastasis involve locoregional lymph nodes, liver, lung, celiac plexus, superior mesenteric vessels, and ligament of treitz. In 1,000 autopsied cases, the most common site for metastases was found to be the liver (63\%), followed by the peritoneum 
$(44 \%)^{[11]}$. The currently reported patient, had documented metastatic disease in the peritoneum which was consistent with known PC metastatic sites, however, the findings noted in the inguinal canal were most unusual. Inguinal hernia is a common finding in the general population ${ }^{[12]}$. FDG PET findings in the region of the inguinal hernia are usually unremarkable (see Figure 2) unless there are complications such as inflammation or infection developing within the herniated bowel loops. Tumors presenting within the inguinal hernia are rare, occurring in less than $0.5 \%$ of surgically excised hernia sacs ${ }^{[13]}$. The differential diagnosis for malignant processes in the inguinal canal includes ovarian carcinomas, mesothelioma, appendiceal adenocarcinomas, pancreatic adenocarcinoma, and rectal adenocarcinoma ${ }^{[14]}$. In a prior study, a SUV cutoff of 2.0 was reported to differentiate between malignant and benign pleural disease with a sensitivity of $91 \%$ and a specificity of $100 \%{ }^{[15]}$. Furthermore, FDG PET was more sensitive and specific than CT (94\% \& $98 \%$ vs. $67 \%$ \& 96\%) in detecting extrahepatic metastases including peritoneal metastases in patients with suspected metastatic or recurrent colorectal adenocarcinoma ${ }^{[16]}$. In addition to increase in FDG uptake in malignancies, other causes include inflammation, post-surgical changes, physiological metabolism, infection, and autoimmune diseases ${ }^{[17]}$. In this patient, the findings in the inguinal canal were unusual for a typical hernia as no bowel loops were noted within the inguinal canal defect. Instead, there was prominent soft tissue stranding associated with increased FDG uptake suggestive of an either inflammatory or a malignant process. Evaluation was recommended for determination of the immediacy of a hernia repair. Surgery was performed 4 days later and the specimen was identified as adenocarcinoma of pancreas primary. It can be inferred that metastasis to the inguinal canal occured via similar mechanism ${ }^{[18]}$. In one study of 35 patients with peritoneal carcinomatosis, CT alone had a sensitivity of $22.2 \%$, specificity of $76.5 \%$, and PPV of $50 \%$. FDG PET and CT had a sensitivity of $66.7 \%$, specificity of $94.1 \%$, and PPV of $92.3 \%$. These results provide important information to the management of patients with peritoneal carcinomatosis ${ }^{[19]}$.

In this patient who has known PC peritoneal metastases, FDG PET imaging demonstrated mild uptake in the peritoneum (SUVmax 3.0) and uptake in the region of the right inguinal hernia (SUVmax 4.0). Although response is speculative without a baseline study, PC metastatic disease in the abdomen is usually successfully visualized by PET/CT ${ }^{[20]}$. With exclusion of the findings in the right inguinal region, in this patient, the aforementioned findings were suggestive of partial response to chemotherapy. However, findings in the IC were consistent with direct involvement of this area by PC metastasizing from the peritoneum to the IC as confirmed by histopathology. Although this finding could not be discerned from an inflammatory process it prompted further evaluation of the patient, by a surgeon, and subsequent surgical intervention. One may surmise that progressing PC has dismal prognosis based on data demonstrating very little progress in improving the current poor response rate with various therapies. Nonetheless, management can still be altered in non-responding patients with more recently approved combination therapies with targeted therapies ${ }^{[21]}$. For example, the median 6-month survival credited to combination therapy was 6.4 months vs. 6.0 months for conventional therapy alone. One-year median survival was increased to $23.8 \%$ from $19.4 \%$ and progression-free survival from 3.5 months to 3.8 months for the combination. Clearly, pancreatic cancer requires additional options that could potentially improve response ${ }^{[21]}$. In the future, the possibility of PC metastasizing to the IC should be recognized by PET/CT interpreting physicians to avoid false interpretations and unnecessary delays in instituting the proper management.

\section{References}

[1] Jemal, A, Siegel, R, Ward, E, Hao, H, Xu, J, Murray, T, et al. Cancer statistics, 2008. CA Cancer J Clin. 2008; 58:71. PMid: 18287387. http://dx.doi.org/10.3322/CA.2007.0010

[2] Chua Yu J, Cunningham David, "Chapter 89. Pancreatic Cancer" (Chapter). Fauci AS, Braunwald E, Kasper DL, Hauser SL, Longo DL, Jameson JL, Loscalzo J: Harrison's Principles of Internal Medicine [Internet]. Available from: http://www.accessmedicine.com/content.aspx?aID=2877991.

[3] Castillo C, Jimenez, Ramon. Epidemiology and risk factors for exocrine pancreatic cancer. In: UpToDate, Basow, DS (17.2), UpToDate, Waltham, MA. 2009.

[4] Steer, M. Clinical manifestations, diagnosis, and surgical staging of exocrine pancreatic cancer. In: UpToDate, Bonis, P (Ed), UpToDate, Waltham, MA. 2009. 
[5] Pakzad F, Grovs AM, Ell PJ: The role of positron emission tomography in the management of pancreatic cancer. Semin Nucl Med. 2006; 36: 248-256. PMid:16762614. http://dx.doi.org/10.1053/j.semnuclmed.2006.03.005

[6] Ozkan H, Kaya M, Cengiz A. Comparison of tumor marker CA 242 with CA 19-9 and carcinoembryonic antigen (CEA) in pancreatic cancer. Hepatogastroenterology 2003; 50: 1669-74. PMid:14571813.

[7] Dewitt J, Devereaux BM, LehmanGA, Sherman S. Imperiale TF: Comparison of endoscopic ultrasound and computed tomography for the preoperative evaluation of pancreatic cancer: a systematic review. Clin Gastroenterol Hepatol. 2006; 4: 717-725; quiz 664. PMid:16675307. http://dx.doi.org/10.1016/j.cgh.2006.02.020

[8] Coombs RJ, Zeiss J, Howard JM, Thomford NR, Merrick HW. CT of the abdomen after the Whipple procedure: value in depicting postoperative anatomy, surgical complications, and tumor recurrence. AJR Am J Roentgenol. 1990; 154: 1011-1014. PMid:2108534.

[9] Mortele KJ, Lemmerling M, Hemptinne B, Vos D, Bock G, Kunnen M. Postoperative findings following the Whipple procedure: Determination of prevalence and morphologicabdominal CT features. Eur Radiol. 2000; 10: 123-128. http://dx.doi.org/10.1007/s003300050017

[10] Li D, Xie K, Wolff R, et al. Pancreatic cancer. Lancet. 2004; 363: 1049-1057. http://dx.doi.org/10.1016/S0140-6736(04)15841-8

[11] Abrams H, Spiro R, Goldstein N. Metastases in Carcinoma: Analysis of 1000 Autopsied Cases. Cancer. 1950 ; 3: 74-85. http://dx.doi.org/10.1002/1097-0142(1950)3:1<74::AID-CNCR2820030111>3.0.CO;2-7

[12] Owens S, Edwards P, Miles K, Allen J. Chronic compartment syndrome affecting the lower limb: MIBI perfusion imaging as an alternative to pressure monitoring: two case reports. Br J Sports Med. 1999; 33(1): 49-51. PMid:10027059 http://dx.doi.org/10.1136/bjsm.33.1.49

[13] Yoell JH. Surprises in hernial sacs: Diagnosis of tumors by microscopic examination. California Med. 1959; 91: 146-148. PMid:13846556

[14] Nicholson P, Donohue J. A Study of Metastatic Cancer Found During Inguinal Hernia Repair. Cancer. 1992; 69(12): 3008-3011. http://dx.doi.org/10.1002/1097-0142(19920615)69:12<3008::AID-CNCR2820691224>3.0.CO;2-8

[15] Benard F, Sterman D, Smith RJ. Metabolic imaging of malignant pleural mesothelioma with fluorodeoxyglucose positron emission tomography. Chest. 1998; 114(3): 713-722. PMid:9743156 http://dx.doi.org/10.1378/chest.114.3.713

[16] Whiteford M, Yee L, Ogunbiyi O, Dehdashti F, Siegel B, Birnbaum E, et al. Usefulness of FDG-PET scan in the assessment of suspected metastatic or recurrent adenocarcinoma of the colon and rectum. Diseases of the Colon\& Rectum. 2000 November; 43(6): 759-767. http://dx.doi.org/10.1007/BF02238010

[17] Abouzied M, Crawford E, Nabi H. 18F-FDG Imaging: Pitfalls and Artifacts. J Nucl Med Technol. 2005 Sep; $33(3): 145-55$. PMid:16145222

[18] Bhosale P, Patnana M, Viswanathan C, Szklaruk J. The Inguinal Canal: Anatomy and Imaging Features of Common and Uncommon Masses. RadioGraphics. 2008; 28: 819-835. PMid:18480486. http://dx.doi.org/10.1148/rg.283075110

[19] Suzuki A, Kawano T, Takahashi N, Lee J, Nakagami Y, Miyagi E, et al. Value of 18F-FDG PET in the detection of peritoneal carcinomatosis. Eur J Nucl Med and Mol Imaging. 2004; 31(12): 1129-31.

[20] Ruf J, Hanninen E, Oettle H, Plotkin M, Pelzer U, Stroszczynski C, et al. Detection of Recurrent Pancreatic Cancer: Comparison of FDG-PET with CT/MRI. Pancreatology. 2005; 5: 266-272. PMid:15855825. http://dx.doi.org/10.1159/000085281

[21] Moore MJ, Goldstein D, Hamm J, Figer A, Hecht J, Gallinger S, et al. Erlotinib plus gemcitabine compared with gemcitabine alone in patients with advanced pancreatic cancer: a phase III trial of the National Cancer Institute of Canada Clinical Trials Group. J Clin Oncol. 2007; 25: 1960-1966. PMid:17452677. http://dx.doi.org/10.1200/JCO.2006.07.9525 